\title{
Ajuste da taxa de câmbio à paridade coberta da taxa de juro no Brasil
}

Exchange rate adjustment to the covered interest rate parity in Brazil

\begin{abstract}
This paper analyzes the adjustment of the covered interest rate parity equation between the Brazilian real and the US dollar. The objective is to evaluate the short-term response of the spot and future exchange rates to shocks in interest rate differential and country risk. The econometric research shows that the adjustment of the covered parity in Brazil occurs with the movement of the spot and future exchange rates in the same direction - an exchange appreciation due to the increase of the interest rate differential and exchange depreciation in the case of the increase of the country risk - but with a higher volatility of the spot exchange rate.
\end{abstract}

\section{Keywords}

covered interest rate parity, exchange rate, future market.

JEL Codes E40, F31, G13.
Pedro Rossi (1)

Eliane Araujo (2)

Nelson H. Barbosa-Filho(3)

(1) Instituto de Economia da Unicamp

(2) Universidade Estadual de Maringá

(3) Escola de Economia de São Paulo

\section{Resumo}

Este artigo analisa o ajustamento da equação da paridade coberta da taxa de juros para a relação entre a moeda brasileira e o dólar americano. $O$ objetivo é avaliar a resposta de curto prazo das taxas de câmbio à vista e futura aos choques no diferencial de juros e no risco país. A investigação econométrica mostra que o ajustamento da paridade coberta no Brasil ocorre com o movimento das taxas de câmbio à vista e futura na mesma direção - de apreciação cambial diante do aumento do diferencial de juros e de depreciação cambial no caso do aumento do risco país -, mas com uma volatilidade maior da taxa de câmbio à vista.

\section{Palavras-chave}

Paridade coberta da taxa de juros, taxa de câmbio, mercado futuro.

Códigos JEL E40, F31, G13. 


\section{Introdução}

O mercado de câmbio brasileiro apresenta como característica, destacada na literatura, uma maior liquidez no mercado futuro do que no mercado à vista. Essa característica tem implicação na eficiência desses mercados e, por conseguinte, no ajustamento das taxas de câmbio à vista e futura. Espera-se que o mercado mais líquido (o mercado futuro) ajuste o preço (taxa de câmbio) com menor volatilidade diante de choques do que o mercado menos líquido (mercado à vista). Esse ajustamento pode ser analisado por meio da paridade coberta da taxa de juros (PCTJ).

A hipótese de paridade coberta de taxa de juro (PCTJ) refere-se à condição teórica que equilibra taxas de juros e taxas de câmbio à vista e a termo, entre duas moedas. Assim a PCTJ estabelece a igualdade que neutraliza a arbitragem com juros e configura a equação de referência para formação dos preços a termo no mercado de derivativos. Mais formalmente, considere um ativo em reais (BRL) e outro em dólares (USD). Expressando todas variáveis em logaritmo, como é usual na literatura, a PCTJ significa que:

$$
i_{U S D}+\sigma+f-s=i_{B R L}
$$

onde $i_{U S D}$ e $i_{B R L}$ são as taxas de juro em USD e BRL, respectivamente, $\sigma$ é o prêmio de risco não cambial, fé a taxa de câmbio futura (forward), e s a taxa de câmbio à vista (spot). Por definição, o período do contrato futuro de câmbio é o mesmo das taxas de juro na equação (1). Com base na PCTJ, a diferença entre a taxa de câmbio futura e à vista deve aumentar sempre que aumentar a diferença entre a taxa de juro doméstica e a taxa de juro externa ajustada pelo risco. Em outras palavras:

$$
f-s=i_{B R L}-\left(i_{U S D}+\sigma\right)
$$

Por exemplo, considere uma elevação da taxa de juro doméstica por razões de controle da inflação, sem nenhuma alteração na taxa de juro externa ou no prêmio de risco não cambial. Nesse caso, a diferença entre a taxa de câmbio futura e à vista $f-s$ deve aumentar, mas existem três formas pelas quais isso pode acontecer:

1) Ajustes em direções diferentes: $f$ sobe e $s$ cai.

2) Ajustes na mesma direção: $f$ e $s$ sobem, mas $f$ sobe mais do que $s$. 
3) Ajustes na mesma direção: $f$ e $s$ caem, mas $s$ cai mais do que $f$. Além disso, essas três formas de ajustamento podem ser acompanhadas de uma volatilidade maior: a) da taxa de câmbio futura ou b) da taxa de câmbio à vista.

Uma breve análise da experiência brasileira indica que o caso 3 é o que acontece na prática. Quando a taxa de juro doméstica sobe em relação à taxa de juro externa ajustada pelo risco, há uma apreciação cambial imediata das taxas de câmbio real/dólar à vista e futura, mas a taxa de câmbio à vista cai relativamente mais, o que aumenta o forward premium. Por analogia, o oposto acontece quando a taxa de juro doméstica cai em relação à taxa de juro externa ajustada pelo risco. Quanto à volatilidade das taxas de câmbio à vista e futura, é esperado que a primeira tenha uma volatilidade maior dada a menor liquidez do mercado.

Nesse contexto, este artigo tem por objetivo comprovar esse resultado intuitivo com base em uma investigação econométrica da evolução da paridade coberta de taxa de juro no Brasil.

Para facilitar a análise, o texto está organizado em quatro seções além desta introdução. Na primeira apresentam-se os principais resultados da literatura sobre a paridade coberta aplicada a taxa de câmbio real/dólar. $\mathrm{Na}$ segunda seção, discorre sobre os aspectos institucionais que condicionam o ajustamento da paridade coberta no Brasil. Já a terceira seção apresenta a metodologia de análise, enquanto a quarta, os resultados do ajustamento da paridade coberta aos choques de juros e risco. Breves considerações finais encerram o artigo.

\section{Principais resultados da literatura}

A literatura internacional sobre a paridade coberta é abundante e geralmente voltada para a verificação de sua validade, usando uma gama variada de modelos estatísticos e de bases de dados. No geral, as evidências empíricas confirmam a validade da paridade coberta, mas também apontam desvios de curto prazo. Frenkel e Levich (1975), por exemplo, confirmam a paridade coberta para a relação entre moedas como o dólar, o macro alemão e a libra e atribuem os desvios a imperfeições do mercado e custos de transação. McCormick (1979) também confirma a paridade coberta, identificando outros motivos para os desvios, como a tributação, 
os controles de capitais e imperfeições na realização de arbitragem. Taylor (1989) e Boulos e Swanson (1994) também identificam importantes desvios da paridade e Baba e Packer (2008) avaliam os efeitos da crise internacional de 2008 sobre paridade coberta euro/dólar por meio de um modelo EGARCH (Exponential Generalized Autoregressive Conditional Heteroscedasticity).

Já na literatura sobre a manifestação da paridade coberta de juros para a relação entre a moeda brasileira e o dólar, destacam-se quatro artigos acadêmicos que fazem uma avaliação empírica da referida relação. Skinner e Mason (2011) avaliam a PCTJ entre diversas moedas, incluindo para o real/dólar, considerando diversas maturidades de juros e taxa futura de câmbio e concluem que a paridade de juros se verifica para prazos curtos (até três meses), mas não para prazos longos quando o tamanho e a frequência das violações da PCTJ são mais importantes, podendo indicar risco de crédito segundo ou autores ou, alternativamente, uma baixa liquidez nos mercados futuros e deficiências na formação da curva de juros para prazos mais longos.

O artigo de Cieplinski, Summa e Braga (2014) confirma a validade da paridade coberta para o período entre 2008 e 2013 e apresenta um conjunto de variáveis alternativas para avaliação da PCTJ contendo o cupom cambial que, segundo os autores, constitui um fechamento tautológico para a paridade coberta. $O$ trabalho também identifica desvios da paridade e corrobora a conclusão de Skinner e Mason (2011), além de não encontrar resultados significativos de validade da paridade coberta para contratos de prazos mais longos.

Neste artigo, para além da confirmação da PCTJ, analisaremos com dados de alta frequência, o seu padrão de ajustamento, ou seja, como os preços futuros e à vista se ajustam diante de choques. Antes disso, na próxima seção tratam-se especificidades da economia brasileira que condicionam o ajustamento da paridade coberta.

\section{Aspectos institucionais da formação da taxa de câmbio no Brasil}

O mercado de câmbio no Brasil tem como característica importante uma liquidez muito maior no mercado futuro do que no mercado à vista. Essa 
característica é enfatizada na literatura, com maior ou menor ênfase, em trabalhos que tratam da institucionalidade do mercado de câmbio brasileiro como os de Prates (2009) e Souza e Hoff (2006), Ventura e Garcia (2009), Dodd e Griffith-Jones (2007), Farhi (2010) e Rossi (2016).

De acordo com Rossi (2016), a diferença regulatória entre os mercados à vista e futuro explica a maior liquidez nesse último mercado:

A formação de posições no mercado de câmbio à vista é restrita a instituições
bancárias e corretoras especializadas, que devem ter autorização do Conselho
Monetário Nacional e suas operações formalizadas em contratos de câmbio e
registradas no SISBACEN. Já no mercado futuro da BM\&F qualquer agente
pode formar posições abertas em dólar futuro, desde que cadastrado no sistema
dessa instituição. As menores exigências regulatórias e a facilidade de acesso
condicionam a atuação dos agentes do mercado de câmbio e atraem investidores
estrangeiros para a negociação da moeda brasileira no mercado futuro (Rossi,
2015, p. 719)'.

Por conta dessa assimetria de liquidez, a taxa de câmbio tende a formar primeiro no mercado futuro e é transmitida por arbitragem para o mercado à vista. Essa é uma visão difundida entre os operadores de mesa de câmbio do mercado financeiro, fato que é tomado como "intuitivo", uma vez que a liquidez se concentra nesse mercado e por conta de o mercado tomar a taxa futura como referência para a cotação dos demais mercados. O trabalho de Ventura e Garcia (2009) identificou que o mercado futuro tem precedência temporal sobre a cotação da taxa de câmbio à vista, defasagens de 10 minutos. Outros autores sustentam a posição de que a taxa de câmbio se forma no futuro, como Franco (2000), Rossi (2016) e Dodd e Griffith-Jones (2007), esses últimos argumentando com base em entrevistas.

Outra característica específica da economia brasileira é uma taxa básica de juros bastante superior à taxa básica de juros internacional ajustada pelo risco para um prazo equivalente. Com livre mobilidade de capitais significa que a taxa de câmbio futura deve estar constantemente acima da taxa de câmbio à vista, de forma a manter a condição dada pela PCTJ. Essa diferença, conhecida como forward premium, está ilustrada na Figura 1.

1 "No mercado de dólar futuro brasileiro não há negociação de dólares. Negocia-se o valor em reais da variação de uma determinada quantidade de dólares em relação à moeda brasileira. Como as operações são liquidadas em reais, não há contratos de câmbio, tampouco movimentação de divisas e, portanto, a legislação cambial, que condiciona a negociação de divisas no mercado à vista, não se aplica a esse mercado" (Rossi, 2015, p. 719-720). 
Figura 1 Dólar à vista e dólar futuro (10 vencimento) no primeiro dia de cada mês de 2016

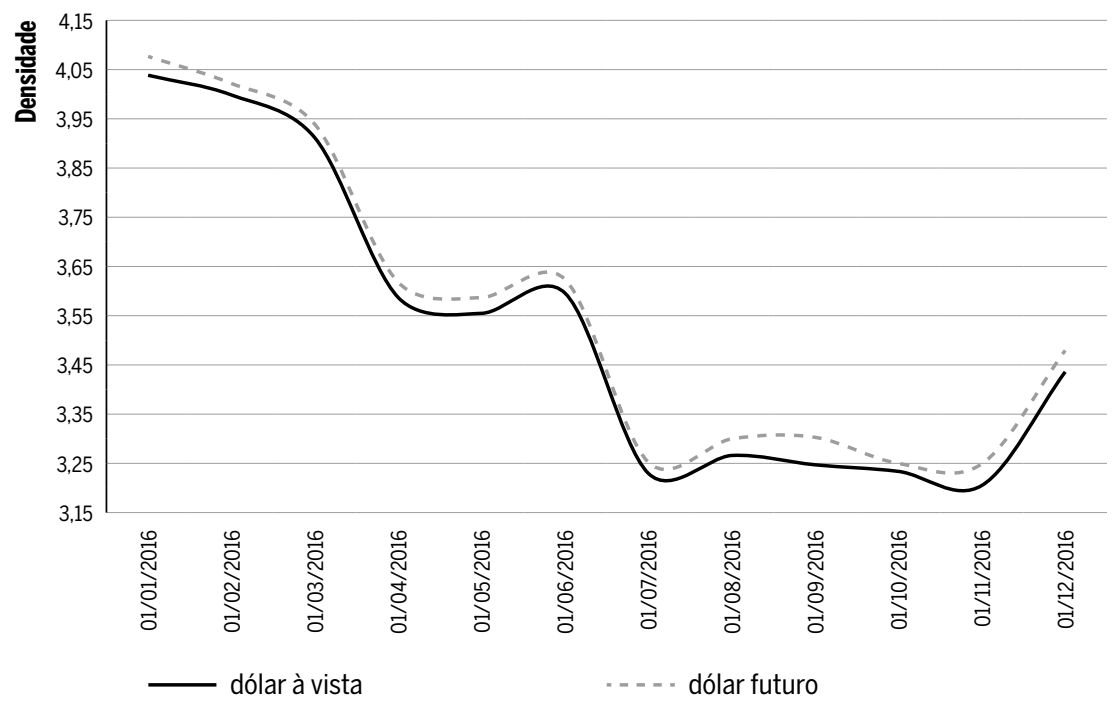

Fonte: Banco Central do Brasil, BM\&F. Elaboração própria.

\section{Dados e metodologia}

Diante dos aspectos institucionais que condicionam a formação da taxa de câmbio no Brasil, nós avaliaremos o padrão de ajustamento das taxas de câmbio à vista e futura em virtude de choques nas variáveis que integram a equação da paridade coberta da taxa de juros. O Quadro 1 apresenta as séries temporais utilizadas nos modelos econométricos em frequência diária.

O período pré-selecionado para a análise da equação de paridade é o que vai de janeiro de 2004 a abril de 2017. A escolha do ano de 2004 para início da análise se deve ao julgamento de que a liquidez dos mercados futuro da BM\&F é suficientemente grande a partir desse ano. Isso porque um mercado futuro com baixa liquidez, como verificado em períodos anteriores, pode afetar o ajustamento da paridade coberta no Brasil.

Destaque-se que o período em questão é marcado por importantes acontecimentos econômicos, como a crise financeira de 2007/2008 e que poderiam implicar quebras estruturais nas séries de dados utilizadas na pesquisa. No entanto, como as séries estão em primeira diferença essas quebras não foram significativas. Isso porque foram aplicados testes de estabilidade 
que examinam se os parâmetros do modelo são estáveis na amostra de dados do modelo (tais como CUSUM e CUSUM of Squares Tests, One-Step Forecast Test, One-Step Forecast Test and One-Step Forecast Test), os quais indicaram que não havia evidências de instabilidade na amostra.

Quadro 1 Séries temporais selecionadas

\begin{tabular}{l|l|l}
\hline Séries & Descrição & Fonte \\
\hline Spot & $\begin{array}{l}\text { 1- Taxa de câmbio - Livre - dólar americano (venda) - } \\
\text { diário - u.m.c./US\$ }\end{array}$ & BCB \\
\hline Futuro & $\begin{array}{l}\text { Taxa de câmbio - real (R\$) / dólar americano (US\$) - } \\
\text { comercial - contrato futuro com vencimento em t+1 }\end{array}$ & IPEADATA \\
\hline Cdi & 4389 - Taxa de juros - CDI anualizada base 252 - \% a.a. & BCB \\
\hline Libor & $\begin{array}{l}\text { Overnight London Interbank Offered Rate (Libor), } \\
\text { based on U.S. Dollar@ (USDONTD156N) }\end{array}$ & Federal Reserve \\
\hline Embi & EMBI+ Risco-Brasil & Ipeadata/JPMorgan \\
\hline CDS Brasil & BRAZIL CDS USD SR 5Y D14 Corp & Bloomberg \\
\hline DXY & dollar index spot (DXY Curncy) & Bloomberg \\
\hline
\end{tabular}

Fonte: Elaboração própria.

No que se refere à metodologia econométrica, como o objetivo da análise é estimar as respostas de curto prazo das taxas de câmbio à vista e futura a choques no diferencial de juros e no risco país, optou-se por estimar um modelo de Vetores Auto Regressivos (VAR) para as primeiras diferenças das variáveis em questão.

Modelos VAR são geralmente utilizados para captar os efeitos de choques aleatórios nas variáveis incluídas no sistema (funções impulso-resposta). ${ }^{2}$ Para um conjunto $\mathrm{K}$ de variáveis de séries temporais, $y_{t}=\left(y_{1 t}, \ldots, y_{k t}\right)$, esse modelo captura as interações dinâmicas entre essas variáveis. Sua forma básica com uma ordem p pode ser representada como segue:

$$
y_{t}=\Sigma_{1}^{p} \Phi_{i} y_{t-1}+u_{t}
$$

onde $\Phi_{i}$ é uma matriz de coeficientes $(\mathrm{K} \times \mathrm{K})$ e $u_{t}=\left(u_{1 t}, \ldots, u_{k t}\right)$ são os termos de erro, supostamente com média zero e independentes. As variáveis introduzidas na equação da paridade coberta dos juros são: 


$$
y_{t}=\left[\begin{array}{c}
\text { Embi }_{t} \\
\text { dif }_{t} \\
\text { futura }_{t} \\
\text { spot }_{t}
\end{array}\right]
$$

Sendo dif o diferencial de juros calculado pela diferença entre a taxa CDI e a libor; spot a taxa de câmbio à vista, futura a taxa de câmbio futura, embi a proxy para o risco, e $t$ o número de defasagens.

Essa ordenação proposta fundamenta-se na decomposição de Cholesky, que é baseada em uma estrutura ordenada de resíduos de forma triangular, o que implica uma estrutura exatamente identificada do modelo. Assim, o método de decomposição de Cholesky supõe uma estrutura de causalidade contemporânea em relação à ordenação das variáveis do sistema, de modo que a primeira variável no ordenamento não é afetada por choques nas demais variáveis, mas choques na primeira variável afetam contemporaneamente as demais variáveis do sistema. A segunda variável é afetada somente pela primeira variável do sistema, mas os choques sobre a segunda variável afetam contemporaneamente as variáveis seguintes, e assim sucessivamente. Dessa forma, as variáveis são ordenadas a partir da variável mais exógena (afetada contemporaneamente apenas pelo próprio choque estrutural) até a variável mais endógena (afetada contemporaneamente por todos os choques).

Além disso, outras variantes serão testadas nessa especificação básica, por exemplo com a inclusão das variáveis CDS e DXY em substituição à variável embi.

\section{Ajustamento da paridade coberta no Brasil}

Com base nos dados e na metodologia descrita, esta seção se propõe a estudar o ajustamento da equação da paridade coberta da taxa de juros para a relação entre a moeda brasileira e o dólar. O objetivo é avaliar a resposta de curto prazo das taxas de câmbio à vista e futura diante de choques no diferencial de taxa de juros e no risco país. A hipótese subjacente é aquela anunciada na introdução: diante de choques, o ajuste das taxas de câmbio à vista e futura ocorre na mesma direção, mas com volatilidade maior no mercado à vista. 
Figura 2 Resposta das taxas de câmbio futuro e à vista e do embi a choques acumulados e não acumulados na variável diferencial de juros

Response to Cholesky One S.D.

Innovations \pm 2 S.E.

\section{Response of DLOGSPOT to DLOGDIF}

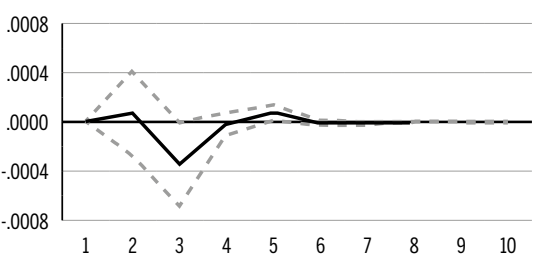

\section{Response of DLOGFUTURO to DLOGDIF}

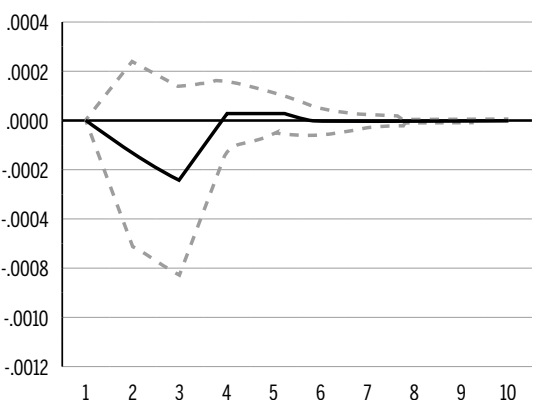

Response of DLOGEMBI to DLOGDIF

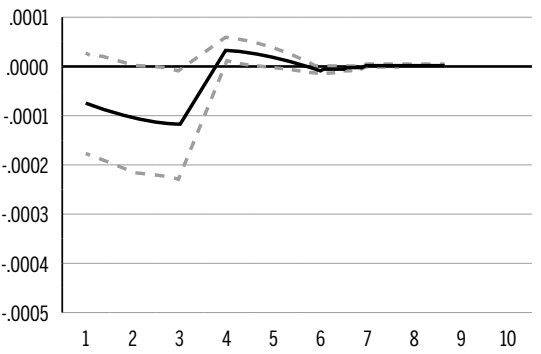

Accumulated Response to Cholesky One S.D.

Innovations \pm 2 S.E.

Accumulated Response of DLOGSPOT to DLOGDIF

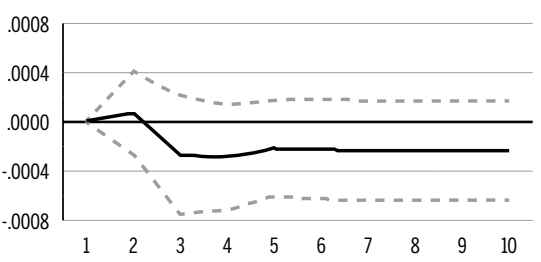

Accumulated Response of DLOGFUTURO to DLOGDIF

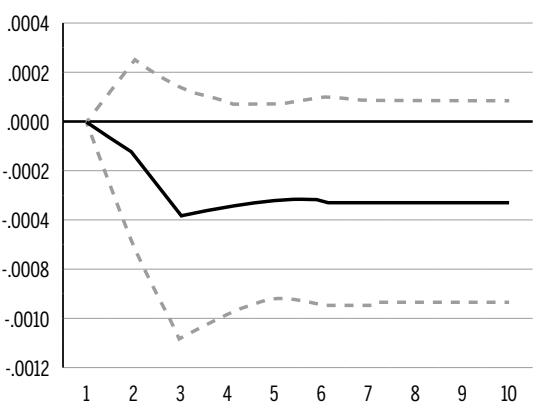

Accumulated Response of DLOGEMBI to DLOGDIF

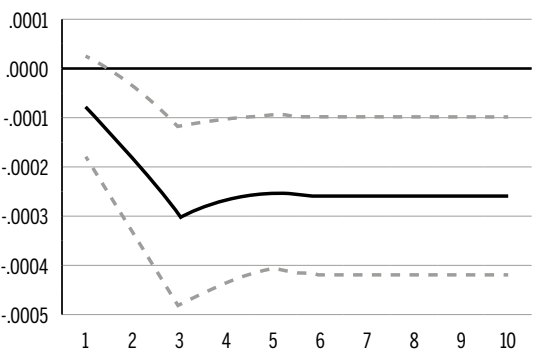

Fonte: Elaboração própria.

Para tanto, as figuras 2 e 3 ilustram as funções impulso-resposta, originadas da estimativa do modelo VAR, com as taxas de variação das variáveis integrantes da equação da paridade coberta dos juros. Consideram-se na Figura 4 os impulsos (choques) na variável diferencial de juros e a resposta 
das taxas de câmbio futura, à vista e do embi.

Figura 3 Resposta das taxas de câmbio futuro e à vista e do diferencial de juros a choque acumulados e não acumulados na variável embi

Response to Cholesky One S.D.

Innovations \pm 2 S.E.

Response of DLOGSPOT to DLOGDIF

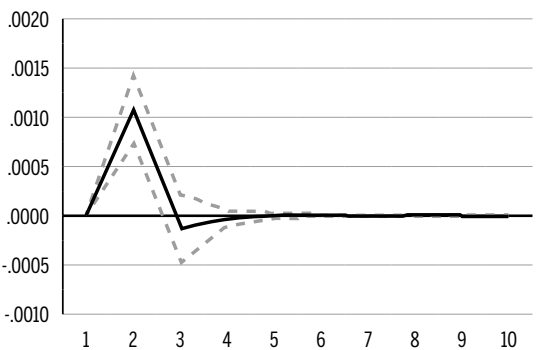

Response of DLOGFUTURO to DLOGDIF

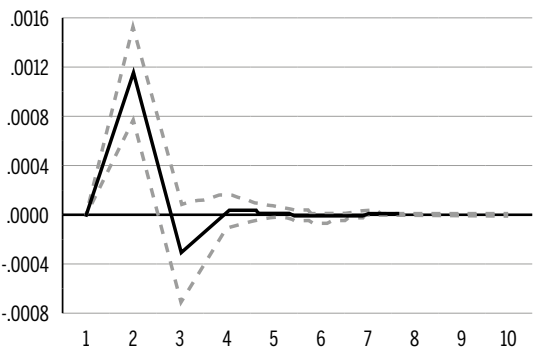

\section{Response of DLOGFUTURO to DLOGDIF}

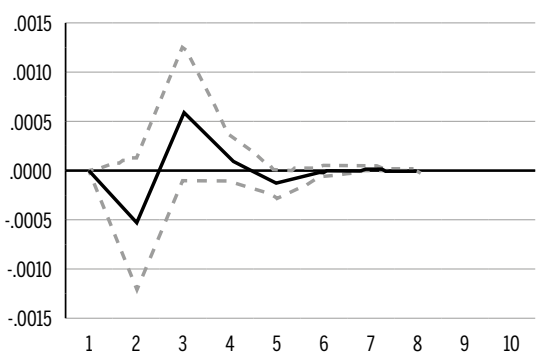

Fonte: Elaboração própria.
Accumulated Response to Cholesky One S.D.

Innovations \pm 2 S.E.

Accumulated Response of DLOGSPOT to DLOGDIF

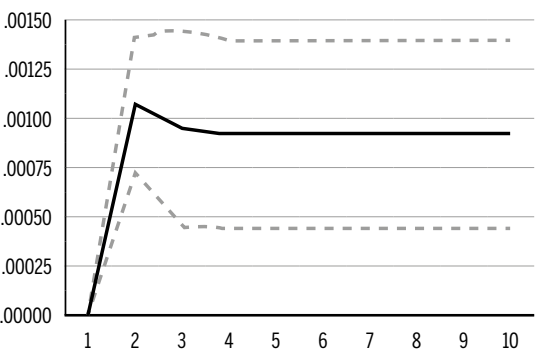

Accumulated Response of DLOGFUTURO to DLOGDIF

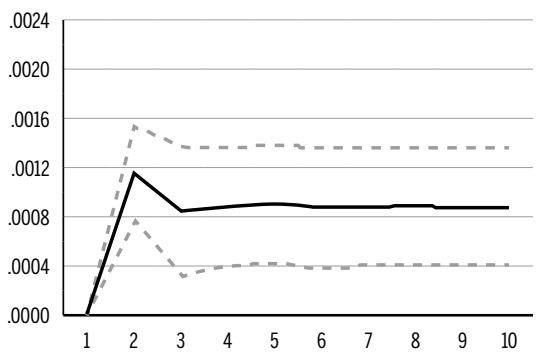

Accumulated Response of DLOGFUTURO to DLOGDIF

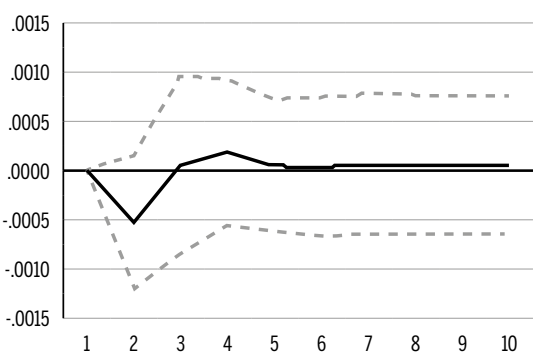


No lado esquerdo da Figura 4 apresentam-se as respostas aos choques não acumulados no diferencial de juros (Figura 4) e no embi. Já o lado direito da Figura 4 ilustra as respostas aos choques acumulados nas mesmas variáveis.

Relativamente à Figura 4 observa-se que um choque positivo no diferencial de juros implica uma apreciação logo no primeiro período na taxa de câmbio futura e, em seguida, no segundo período, na taxa de câmbio à vista. A proxy para o risco país, isto é, o embi também responde negativamente à elevação no diferencial de juros.

No que se refere às respostas acumuladas, uma elevação no diferencial de juros leva, como esperado, a um patamar de taxas de câmbio futura e à vista menor, ou mais apreciada, quando comparado ao nível prevalecente antes do choque. $\mathrm{O}$ efeito acumulado no risco país também é negativo, dado um choque positivo no diferencial de juros o embi passa a situar-se em patamar inferior ao que prevalecia anteriormente.

Figura 4 Ajustamento das taxas de câmbio futuro e à vista a choque acumulados e não acumulados em dif e embi

\section{choque em dif}

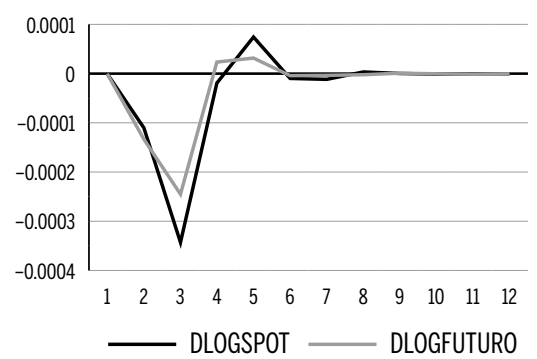

choque em embi

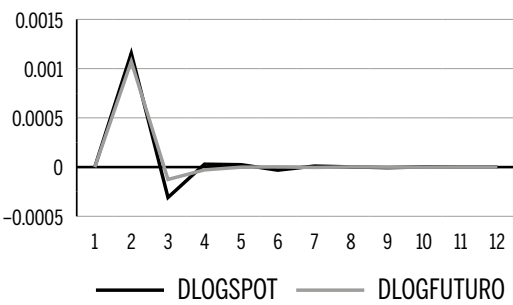

\section{choque acumulado em dif}

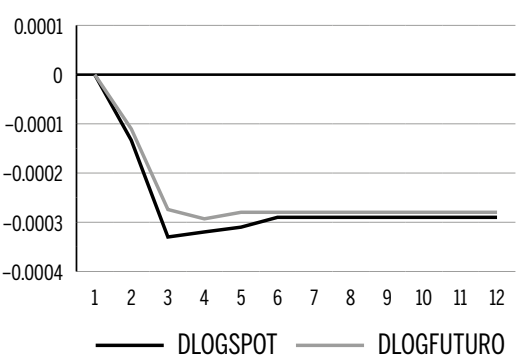

choque acumulado em embi

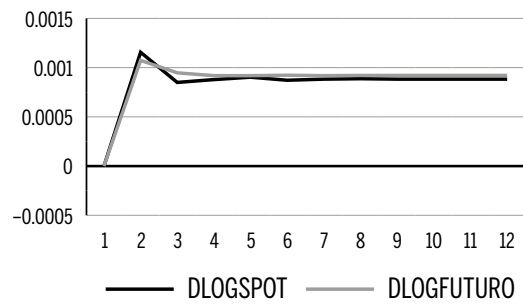

Fonte: Elaboração própria. 
Considerando ainda a Figura 4, observa-se que um choque positivo na variável embi ocasiona elevação imediata nas taxas de câmbio futura e à vista que se inicia logo no primeiro período. $O$ diferencial de juros, apesar de um leve movimento de queda inicial, se eleva em decorrência do choque positivo no risco país. Como esperado, os efeitos acumulados desse choque são níveis mais elevados, ou depreciados, das taxas de câmbio à vista e futura e um patamar levemente superior da variável diferencial de juros.

Por fim, a Figura 4 permite comparar o ajustamento das taxas à vista e futura diante dos choques de aumento do diferencial de juros e do aumento do risco país. Nota-se que o ajustamento da taxa de câmbio à vista ocorre de maneira mais volátil do que a taxa de câmbio futura, tanto no caso do choque de diferencial de juros, quanto no choque de risco país. Corrobora-se, portanto, a hipótese inicial desta pesquisa.

A análise do efeito acumulado do choque de diferencial de juros e do aumento do risco (Figura 4) permite identificar também um aumento do forward premium. ${ }^{3}$ Isso porque, diante de um aumento do diferencial de juros, a resposta acumulada do câmbio à vista indica uma apreciação cambial maior dessa variável em relação à taxa de câmbio futura, identificável na Figura 4, o que aumenta a diferença entre as duas taxas e o forward premium. Menos perceptível graficamente, porém corroborado pelos dados da função impulso resposta, diante do choque de risco país a taxa de câmbio futura (linha laranja) deprecia mais do que a taxa de câmbio à vista (linha azul), aumentando a diferença entre as duas taxas e o forward premium. Assim, o modelo capta um novo equilíbrio de ajustamento da paridade coberta, diante das mudanças dos parâmetros de diferencial de juros e de risco.

Vale dizer que, após a estimação do modelo VAR, foram realizados os testes usuais de robustez, apresentados no anexo deste artigo. Destaque-se que o modelo foi estimado com duas defasagens. Também outras variáveis foram incluídas na equação de paridade, outras proxies para o risco país como o CDS e o DXY, no entanto, essa mudança de especificação não implicou alterações nos resultados centrais apresentados.

3 Quanto maior o diferencial de juros e o risco país, maior é o forward premium e quanto maior o forward premium, maior é a distancia entre as taxas spot e futura. No caso do aumento do diferencial de juros, a distância entre o spot e o futuro aumenta por conta da valorização mais intensa da taxa spot com relação à taxa futura. Já no caso do aumento do risco país, a diferença entre as duas taxas aumenta por conta da desvalorização maior da taxa futura em relação à taxa spot. 


\section{Considerações finais}

Este artigo mostrou que o ajustamento da paridade coberta no Brasil ocorre com o movimento das taxas de câmbio à vista e futura na mesma direção, mas com uma volatilidade maior da taxa de câmbio à vista. Essa conclusão decorre da análise das funções impulsos respostas do modelo VAR diante dos choques de aumento de diferencial de juros e de aumento de risco país. No primeiro caso, o efeito acumulado do choque é de apreciação das taxas de câmbio à vista e futura, mas com maior volatilidade da taxa de câmbio à vista. Já no caso de aumento de risco país, o efeito acumulado é de depreciação cambial, mais uma vez, com maior volatilidade da taxa de câmbio à vista. Em ambos os casos, no novo equilíbrio de ajustamento da paridade coberta há um forward premium maior, como esperado.

Esse resultado provavelmente decorre das características institucionais do mercado de câmbio brasileiro que apresenta uma liquidez muito maior no mercado futuro do que no mercado à vista. A menor volatilidade do mercado futuro também é indicativa de que a taxa de câmbio se forma primordialmente nesse mercado.

Por fim, a avaliação do ajustamento da paridade coberta no Brasil, para além de lançar luz sobre a eficiência dos mercados de câmbio, também pode ser relevante para o entendimento da formação da taxa de câmbio real/dólar e para o processo de tomada de decisão de política cambial. A assimetria entre os mercados futuro e o mercado à vista de câmbio implica efeitos distintos das políticas cambiais voltadas para o mercado à vista, como as intervenções e os controles de fluxos de capitais, daquelas destinadas ao mercado futuro, como os swaps e a regulação de operações com derivativos cambiais. Os efeitos dessas políticas podem ser estudados considerando a dinâmica de ajustamento da taxa de câmbio discutida neste artigo.

\section{Referências}

BABA, N.; PACKER, F. Interpreting deviations from covered interest parity during the financial market turmoil of 2007-2008. Journal of Banking \& Finance, 2008.

BOULOS, N.; SWANSON, P. Interest rate parity in times of turbulence: the issue revisited. Journal of Financial and Strategic Decisions, v. 7, n. 2, 1994. 
CIEPLINSKI, A.; SUMMA, R.; BRAGA, J. Avaliação empírica do teorema da paridade coberta para a economia brasileira. IE UFRJ. Texto para discussão, n. 022, 2014.

DODD, R.; GRIFFITH-JONES, S. Brazil's derivatives markets: hedging, Central Bank intervention and regulation. Economic Commission for Latin America and the Caribbean (Eclac), 2007.

FARHI, M. Os impactos dos derivativos no Brasil. In: MARCOLINO, L. C.; CARNEIRO, R. Sistema financeiro e desenvolvimento no Brasil. São Paulo: Atitude, 2010.

FRANCO, G. The real plan and exchange rate. Essays International Finance, Princeton University, n. 216, 2000.

FRENKEL, J. A.; LEVICH, R. M. Covered interest arbitrage: unexploited profits? The Journal of Political Economy, p. 325-338, 1975.

HAMILTON, J. D. Time series analysis. Princenton: Princeton University Press, 1994.

McCORMICK, F. Covered interest arbitrage: Unexploited profits? Comment. The Journal of Political Economy, p. 411-417, 1979.

PRATES, D. M. Os determinantes das taxas de câmbio nominal e real no Brasil no período 2003-2007. In: FERREIRA, F.; MEIRELES, B. Ensaios sobre economia financeira. Rio de Janeiro: BNDES, 2009.

ROSSI, P. Política cambial no Brasil: um esquema analítico. Revista de Economia Política, 35 (4), 2015, pp. 708-727, 2015.

ROSSI, P. Taxa de câmbio no Brasil: teoria, institucionalidade, papel da arbitragem e da especulação. São Paulo: Editora FGV, 2016.

SKINNER, F. S.; MASON, A. Covered interest rate parity in emerging markets. International Review of Financial Analysis, v. 20, n. 5, p. 355-363, 2011.

SOUZA, F. E. P.; HOFF, C. R. O regime cambial brasileiro: sete anos de flutuação. In: BERLINSKI, J. et al. (Org.). 15 años de Mercosur. Montevidéu, Zonalibro, 2006, 10, n. 3, 2009.

TAYLOR, M. P. Covered interest arbitrage and market turbulence. The Economic Journal, p. 376-391, 1989.

VENTURA, A.; GARCIA, M. Mercados futuro e à vista de câmbio no Brasil: o rabo balança o cachorro. Texto para discussão. PUC-Rio, n. 563, 2009.

\section{Sobre os autores}

Pedro Rossi-pedrolrossi@gmail.com

Professor do Instituto de Economia, Universidade Estadual de Campinas, Campinas, São Paulo, Brasil.

ORCID: https://orcid.org/0000-0002-2504-9922.

Eliane Araujo - elianedearaujo@gmail.com

Professora da Universidade Estadual de Maringá, Maringá, Paraná, Brasil.

ORCID: https://orcid.org/0000-0001-5104-2508.

Nelson H. Barbosa-Filho-nhbarbosa@gmail.com

Escola de Economia de São Paulo, Fundação Getúlio Vargas, São Paulo, SP, Brasil.

Universidade de Brasília, Brasília, Distrito Federal, Brasil.

ORCID: https://orcid.org/0000-0003-3889-5703.

\section{Sobre o artigo}

Recebido em 08 de março de 2018. Aprovado em 04 de fevereiro de 2019. 


\section{APÊNDICE}

Os testes de robustez habituais foram aplicados. Inicialmente, verificamos a autocorrelação nos resíduos do modelo. Não há evidências para rejeitar a hipótese nula (inexistência de autocorrelação serial) após a inclusão da segunda defasagem no modelo (Tabela A1). Já Tabela A2 destaca as evidências contra a rejeição da hipótese nula de que os resíduos são homocedásticos, indicando a inexistência de heteroscedasticidade.

Tabela A1 VAR Residual Serial Correlation LM Tests

Null Hypothesis: no serial correlation at lag order $\mathrm{h}$

Included observations: 3006

\begin{tabular}{l|r|r}
\hline Lags & LM-Stat & Prob \\
\hline 1 & 54,31217 & 0,0000 \\
\hline 2 & 28,83093 & 0,1210 \\
\hline
\end{tabular}

Tabela A2 VAR Residual Heteroskedasticity Tests

Null Hypothesis: no Heteroskedasticity

Included observations: 3006

\begin{tabular}{l|r|r}
\hline Joint test: & df & Prob \\
\hline Chi-sq & 800 & 0,2171 \\
\hline 831,0126 & & 80 \\
\hline
\end{tabular}

O teste de normalidade de Jarque-Bera sugere a rejeição da hipótese de que erros seguem uma distribuição normal (Tabela A3). No entanto, esse problema pode ser minimizado seguindo o Teorema do Limite Central, segundo o qual à medida que o tamanho da amostra de uma dada variável aumenta, a amostra média de distribuição tenderá ao normal.

Tabela A3 VAR Residual Normality Tests

Orthogonalization: Cholesky (Lutkepohl)

Null Hypothesis: residuals are multivariate normal

\begin{tabular}{lr|r|r}
\hline Component & Jarque-Bera & df & Prob \\
\hline $\mathbf{1}$ & 13822,16 & 2 & 0,0000 \\
\hline $\mathbf{2}$ & 29303333 & 2 & 0,0000 \\
\hline $\mathbf{3}$ & 8963080 & 2 & 0,0000 \\
\hline Joint & 38288918 & 8 & 0,0000 \\
\hline
\end{tabular}


Por fim, verificamos a estabilidade do modelo. De acordo com a Figura A1, todas as raízes inversas do polinômio característico AR estão dentro do círculo unitário, o que significa que o sistema VAR é estável. Em suma, os testes de robustez indicam que no modelo estimado (com duas defasagens) os resíduos são não correlacionados e homocedásticos, apesar de não serem normais, bem como indicam que o sistema é estável.

\section{Figura A1 Teste de estabilidade}

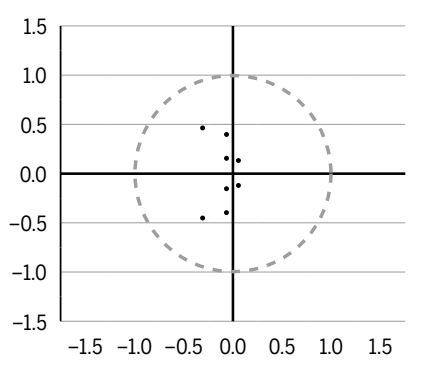

